\title{
Lentiviral vector-mediated survivin shRNA delivery in gastric cancer cell lines significantly inhibits cell proliferation and tumor growth
}

\author{
RAEES HABIB $^{1 *}$, JAVED AKHTAR $^{2 *}$, MOHAMMAD TAQI $^{3}, \mathrm{CHE} \mathrm{YU}^{4}$ and CHUNQING ZHANG ${ }^{1}$
}

Departments of ${ }^{1}$ Gastroenterology and ${ }^{2}$ Thoracic Surgery, Provincial Hospital Affiliated to Shandong University, Jinan, Shandong 250021; ${ }^{3}$ Department of General Surgery, Qilu Hospital of Shandong University, Jinan, Shandong 250012;

${ }^{4}$ Department of Nephrology, Provincial Hospital Affiliated to Shandong University, Jinan, Shandong 250021, P.R. China

Received March 6, 2015; Accepted April 9, 2015

DOI: 10.3892/or.2015.4033

\begin{abstract}
It has been well documented that survivin has multiple functions including cytoprotection, inhibition of cell death, and cell cycle regulation, particularly at the mitotic stage of the cell cycle, all of which favor cancer survival. Its expression in normal tissue is developmentally regulated, and any type of deregulation in survivin expression favors cancer survival. Gastric cancer is one of the most common malignancies and the second most common cause of cancer-related mortality worldwide. The molecular mechanisms involved in the transformation and progression of gastric cancer remain unclear. In the present study, we investigated the effect of lentiviral vector-mediated survivin shRNA delivery in gastric cancer cell lines. Lentiviral-mediated survivin shRNA was used to knock down survivin expression in gastric cancer cell lines SGC-7901, MGC-803 and MKN-28. The Transwell chemotaxis and the CCK- 8 assays were used to assess the migration and proliferation of the tumor cells, respectively. TUNEL assay was used to detect apoptosis. Quantitative real-time PCR and western blot analysis were used to quantify mRNA and protein levels, respectively. Our results demonstrated that lentiviral-mediated RNAi markedly suppressed the survivin expression in all three gastric cancer cell lines. Significant decrease in survivin mRNA and protein expression were detected in the gastric cancer cell lines stably transfected with the lentiviral survivin shRNA vector, and knockdown of survivin also significantly inhibited the proliferation and migration in the gastric cancer cells and tumorigenicity in a
\end{abstract}

Correspondence to: Professor Chunqing Zhang, Department of Gastroenterology, Provincial Hospital Affiliated to Shandong University, 324 Jingwu Weiqi Road, Jinan, Shandong 250021, P.R. China

E-mail: zhchqing@medmail.com.cn

${ }^{*}$ Contributed equally

Key words: gastric cancer, survivin, biological markers, shRNA xenograft animal model. Our results indicated that aberrant high cytoplasmic survivin expression in gastric cancer cells is associated with increased proliferation index and tumor growth. In conclusion, our results suggest that lentiviralmediated gene therapy has the potential to be developed into a novel therapeutic strategy for the treatment of gastric cancer.

\section{Introduction}

Gastric cancer is one of the most common cancers worldwide (1). Approximately one million new cases of stomach cancer were estimated to have been diagnosed in 2008 (988,000 cases, $7.8 \%$ of the total), making it currently the fourth most common malignancy in the world, behind cancers of the lung, breast and colorectum. More than $70 \%$ of cases (713,000 cases) occur in developing countries $(467,000$ in males and 246,000 in females) and half of the world total occurs in Eastern Asia (mainly in China). The age of onset for developing gastric cancer in the Chinese population is younger than that in the West. Approximately 21,600 patients are diagnosed annually in the United States, of whom 10,990 are expected to succumb to the disease (2). Surgical treatment remains the best treatment option for potential cure yet only for selected patients suitable for surgery. Given the poor prognosis and the fact that most gastric cancer cases are diagnosed at the advanced or unresectable stage, new therapeutic strategies, treatment options and novel therapeutic targets are desperately needed. Drug development has been transformed following the identification and ability to direct treatment at specific molecular targets.

Survivin, also called baculoviral inhibitor of apoptosis repeat-containing 5 or BIRC 5 , is a protein that, in humans, is encoded by the BIRC5 gene. Survivin is a member of the inhibitor of apoptosis (IAP) family which encodes negative regulatory proteins that prevent apoptotic cell death. IAP family members usually contain multiple baculovirus IAP repeat (BIR) domains, but this gene encodes proteins with only a single BIR domain. The encoded proteins also lack a C-terminus RING finger domain. Survivin has multiple functions including cytoprotection, inhibition of cell death, and cell cycle regulation, particularly at the mitotic stage of the 
cell cycle, all of which favor cancer survival (3). Survivin plays critical roles in cell division and cell survival $(4,5)$. IAP and BCL-2 family proteins are critically important for the regulation of apoptosis (6). Increased survivin mRNA or protein expression has previously been reported to be a prognostic indicator of tumor progression in different types of human cancer (7-12). Cytoplasmic expression of survivin is common in breast cancer and may be a useful clinical diagnostic and prognostic marker (13). Survivin, an apoptosis inhibitor/cell-cycle regulator, is critically required for suppression of apoptosis and ensuring normal cell division in the $\mathrm{G} 2 / \mathrm{M}$ phase of the cell cycle (14). In cancer cells, elevated survivin is commonly associated with an enhanced proliferative index, reduced level of apoptosis, resistance to chemotherapy, and increased rate of tumor recurrence (15-18). More recently, survivin expression was also shown to enhance the metastatic potential of cancer cells by promoting, together with XIAP, NF- $\kappa$ B-dependent transcription and secretion of fibronectin (19). Survivin expression has been reported to be low in normal tissues.

RNA interference (RNAi) is one of the most important technological breakthroughs in modern biology, allowing the direct observation of the effects of the loss of function of specific genes in mammalian systems. Recent studies advocate RNAi as a promising therapeutic tool against infectious diseases and cancer (20). Our recent research demonstrated that lentiviral vector-mediated delivery of short hairpin RNA (shRNA) resulted in the persistent knockdown of gene expression $(21,22)$. A previous study reported that knockdown of survivin expression in melanoma utilizing siRNA inhibited cell proliferation and sensitized the cells to apoptosis induced by chemotherapy (23). However, stable suppression of survivin expression by RNA remains to be investigated.

In the present study, we assessed the feasibility of lentiviral vector-delivered shRNA against survivin for the treatment of gastric cancer in vitro and in an experimental animal model. Lentiviral-mediated survivin shRNA was utilized to knock down survivin expression in gastric cancer cell lines (SGC7901, MGC-803 and MKN-28). The results showed that silencing of survival expression inhibited cell proliferation and tumor growth.

\section{Materials and methods}

Mice, cell lines, culture medium and reagents. BALB/C-nu-nu male mice (weight 18-22 g, 6-weeks old) and human gastric cancer cell lines SGC-7901, MGC-803, MKN-28 and human embryonic kidney 293 cells (HEK93T) were obtained from the Shanghai Tumor Institution. Classical Liquid Media/ Dulbecco's modified Eagle's medium (DMEM) and high glucose RPMI-1640 media were purchased from HyClone (Thermo Fisher Scientific, Waltham, MA, USA). Fetal bovine serum (FBS) was purchased from Gibco (Invitrogen, Carlsbad, CA, USA). Dimethyl sulfoxide (DMSO) was purchased from Sigma (St. Louis, MO, USA). Rabbit anti-survivin polyclonal antibody and HRP-conjugated secondary antibodies were purchased from Abcam (Cambridge, MA, USA).

All experimental procedures using animals in the present study had received prior approval by the Institutional Animal Care and Use Committee of Shandong University under contract 2011-0015.
Cell culture. The SGC-7901, MGC-803 and MKN-28 cell lines were grown in RPMI-1640 medium supplemented with $10 \% \mathrm{FBS}$ and $100 \mathrm{U} / \mathrm{ml}$ penicillin and $100 \mathrm{mg} / \mathrm{ml}$ streptomycin (Gibco, Invitrogen). The cells were grown at $37^{\circ} \mathrm{C}$ in a humidified atmosphere containing $5 \% \mathrm{CO}_{2}$. Stock cultures of each cell line were routinely sub-cultured at least once a week, and the medium was changed every 2-3 days.

Immunocytochemistry. Stably transfected gastric cancer cell lines were seeded into 4-chambered glass slides (Nunc Lab-Tek Chamber Slide System). The cells were then incubated overnight. After $24 \mathrm{~h}$, the cells were rinsed with PBS, fixed with $3.7 \%$ w/v paraformaldehyde (Sigma), rinsed with PBS and permeablized in $0.5 \%$ Triton X-100 (Sigma). Nonspecific immunoglobulin binding was blocked with 5\% normal goat serum and $0.5 \%$ NP-40 (Sigma). Primary antibodies recognizing survivin (Abcam) were diluted 1:100 in blocking solution. After incubation with the primary antibody, the cells were rinsed with $0.05 \%$ Tween-20 (Bio-Rad, Hercules, CA, USA) in PBS, and then incubated with a secondary antibody for $1 \mathrm{~h}$ at room temperature. Staining with 3,3'-diaminobenzidine (DAB) was carried out and observed under a light microscope.

Lentiviral shRNA vector constructs. pGIPZ-lentiviral shRNAmir vectors targeting the human survivin gene and the non-silencing pGIPZ control vector were purchased from Open Biosystems (Thermo Fisher Scientific). pGIPZ non-silencing control vector was used as expression control to generate non-silencing lentiviral stock to optimize expression conditions in the mammalian cell line of interest. pGIPZ cloning vector containing Turbo GFP reporter and also elements were required to allow packaging of the expression construct into virions (such as 5' and 3 ' LTRs and $\Psi$ packaging signal). pGIPZ vector also expresses a puromycin-resistant gene. The sequences of survivin shRNA were: TCTTGA ATGTAGAGATGCG, TTCCTAAGACATTGCTAAG and AGCAGAAGAAACACTGGGC. 21-mer oligonucleotide TCTCGCTTGGGCGAGAGTAAG that had no significant homology to any known human mRNA in the databases was cloned in the same vector and used as the control.

Virus production and transduction. Lentiviral shRNA was produced by co-transfection of the Trans-Lentiviral packaging mix with a shRNA transfer vector into HEK $293 \mathrm{~T}$ packaging cells (Open Biosystems). The packaging mix contains an optimized mixture of five packaging plasmids (pTLA1-Pak, pTLA1-Enz, pTLA1-Env, pTLA1-Rev and pTLA1-TOFF) to facilitate viral packaging of the transfer vector following co-transfection into HEK293T producer cells. HEK293T cells stably express the SV40 large T antigen (simian vacuolating viral particles $40 \mathrm{TAg}$ ) which allows high levels of protein to been expressed from vectors containing the SV40 origin of replication. Following co-transfection, replication-incompetent virions were released into the media which were collected after 48 and $72 \mathrm{~h}$, respectively. For cell infection, viral supernatants were supplemented with $6 \mu \mathrm{g} / \mathrm{ml}$ polybrene and incubated with the cells for $24 \mathrm{~h}$. Gastric cancer cell lines were transduced by the lentiviral particles followed by puromycin selection $(1 \mu \mathrm{g} / \mathrm{ml})$ for 10 days. The cell lines stably expressing shRNA were maintained in puromycin $(0.2 \mu \mathrm{g} / \mathrm{ml})$. 
$R N A$ extraction and $q R T-P C R$. Total RNA extraction was performed using TRIzol reagent (Invitrogen) according to the manufacturer's instructions. RNA concentration was measured by NanoDrop 1000 (Thermo Fisher Scientific). One microgram of total RNA extracted from the cells was subjected to reverse transcription (RT). The Verso cDNA kit (Thermo Fisher Scientific) was used for cDNA synthesis. Real-time RT-PCR was used to quantify the expression level of the survivin gene in the gastric cancer cell lines SGC-7901, MGC-803 and MKN-28 using ABI 7300 real-time PCR thermal cycle instrument (ABI, USA), according to the supplied protocol. Amplification conditions were as follows: reverse-transcription reaction: $42^{\circ} \mathrm{C}, 30 \mathrm{~min} /$ cycle. $\mathrm{PCR}$ cycling conditions were as follows: enzyme activation $95^{\circ} \mathrm{C}, 15 \mathrm{~min} /$ cycle; denaturation $95^{\circ} \mathrm{C}, 15 \mathrm{sec} / 40$ cycles and annealing/extension at $60^{\circ} \mathrm{C}$ for $60 \mathrm{sec}$.

A real-time PCR reaction was performed using the Solaris qPCR Gene Expression Master Mix with Low ROX premixed and $1 \mu l$ of total cDNA in each well, Survivin-specific primers were: forward, ACCGCATCTCTACATTCAAG and reverse, CAAGTCTGGCTCGTTCTC. The relative expression levels were normalized to the expression of endogenous $\beta$-actin. Primers were forward, TCACCCACACTGTGCCCATCTA CGA and reverse, CAGCGGAACCGCTCATTGCCAATGG.

Protein extraction and western blot analysis. For whole-cell protein extraction, the cells were washed with cold PBS and subsequently lysed in cold RIPA lysis buffer [50 mM Tris- $\mathrm{HCl}$, $\mathrm{pH} 7.4,150 \mathrm{mM} \mathrm{NaCl}, 1 \mathrm{mM}$ dithiothreitol (DTT), $0.25 \%$ sodium deoxycholate, $0.1 \% \mathrm{NP}-40$ ] containing $1 \mathrm{mM}$ phenylmethysulfonyl fluoride (PMSF), $50 \mathrm{mM}$ sodium pyrophosphate, $1 \mathrm{mM} \mathrm{Na} \mathrm{VO}_{4}, 1 \mathrm{mM} \mathrm{NaF}, 5 \mathrm{mM}$ EDTA, $5 \mathrm{mM}$ EGTA and a protease inhibitor cocktail (Roche Diagnostics, Mannheim, Germany). Cell lysis was performed on ice for $30 \mathrm{~min}$. Clear protein extracts were obtained by centrifugation for $30 \mathrm{~min}$ at $4^{\circ} \mathrm{C}$. Protein concentrations were determined by the method of Bradford using the Bio-Rad protein assay reagent (Bio$\mathrm{Rad}$ ) and 20-40 $\mathrm{mg}$ of protein mixed with loading buffer was loaded per lane, and separated by $12 \%$ SDS-polyacrylamide gel electrophoresis (SDS-PAGE). Proteins were transferred to PVDF membrane filters (Millipore, Billerrica, MA, USA). Nonspecific binding was blocked by incubation in phosphatebuffered saline (PBS) containing 0.1\% Tween-20 (PBS-T) and $5 \%$ skim milk. PVDF membranes were blocked with $5 \%$ dry milk for $1 \mathrm{~h}$ at $4^{\circ} \mathrm{C}$. Membranes were incubated with the survivin primary antibody $(1: 2,000)$ overnight at $4^{\circ} \mathrm{C}$. The membranes were then incubated with the corresponding secondary antibody $(1: 2,500$, horseradish peroxidase-conjugated anti-rabbit) in TBST-5\% nonfat milk for $1 \mathrm{~h}$ at room temperature and the immunoreactive bands were visualized using the EZ ECL chemiluminescence detection kit for HRP (Biological Industries Ltd., Kibbutz Beit Haemek, Israel). Images were acquired using the LAS3000 Imager (Fujifilm, Tokyo, Japan). Membranes were re-probed for $\beta$-actin as a loading control.

Cell proliferation assay. The Cell Counting Kit-8 (CCK-8; Dojindo, Kunamoto, Japan) was used for the cell proliferation assay. Five thousand viable cells/well were placed into 96 -well tissue culture plates in a final volume of $100 \mu \mathrm{l}$. Every $24 \mathrm{~h}$, a plate was subjected to assay by adding $10 \mu \mathrm{l}$ of CCK-8 solution to each well, and the plate was further incubated for $4 \mathrm{~h}$ at $37^{\circ} \mathrm{C}$. The absorbance at $450 \mathrm{~nm}$ was measured with a microplate reader. The experiment was performed in 10 replicates.

Migration and invasion assays. For the Transwell migration assay, 30,000 cells were added to the upper chamber in serumfree media and migration at $37^{\circ} \mathrm{C}$ towards $10 \%$ FBS containing growth media was determined either after 24 or $48 \mathrm{~h}$. The cells that migrated through the membrane were fixed, stained with H\&E (Sigma) and counted under a light microscope. For the invasion assay, lower chambers of Matrigel-coated invasion plates were coated with $10 \mathrm{mg} / \mathrm{ml}$ fibronectin overnight at $4^{\circ} \mathrm{C}$, and cells invading through the Matrigel were fixed and stained after $48 \mathrm{~h}$.

TUNEL assay. Terminal deoxynucleotidyl transferase-mediated dUTP nick end labeling (In Situ Cell Death Detection kit, POD) was used for confirming apoptosis of individual cells. The TUNEL working procedure was carried out following the manufacturer's instructions (Roche). Endogenous peroxidase was blocked by incubation in $1.3 \% \mathrm{H}_{2} \mathrm{O}_{2}$ in PBS for $10 \mathrm{~min}$ at room temperature before enzymatic labeling. During the TUNEL procedure, the samples were washed in PBS. The fluorescent signal conversion using anti-fluorescence antibody conjugated with peroxidase and substrate color reaction applying chromogen DAB (3,3'-diaminobenzidine tetrahydrochloride; Sigma-Aldrich, Steinheim, Germany) were carried out after enzymatic labeling.

In vivo studies of gastric cancer xenograft tumor models in nude mice. Six-week-old male BALB/c nude mice were housed in a temperature-controlled, pathogen-free animal facility with a 12-h light and dark cycle. The mice were injected subcutaneously into bilateral flanks with untransfected cells, or cells transfected with the non-silencing shRNA or survivin shRNA $\left(2 \times 10^{6}\right.$ cells in $200 \mu \mathrm{l}$ PBS $)$ to establish tumors. The tumor mass (xenograft) volume was measured every week from week 3 to 7 . After week 7, the mice were sacrificed and tumors were harvested.

Statistical analysis. For comparison of more than three groups, one-way analysis of variance was used, followed by Tukey's multiple comparison. P-values $<0.05$ were considered to indicate statistically significant results. One-way analysis of variance (ANOVA), followed by the LSD post hoc test was used to compare mean differences in two or more groups. All statistical analysis was performed using IBM SPSS version 20.0 .

\section{Results}

Survivin is highly upregulated in the gastric cancer cell lines. We assessed the survivin gene expression in the gastric cancerderived cell lines by immunocytochemistry (ICC) (Fig. 1). Strong immunoreactivity of survivin protein was detected in the cytoplasm of the gastric cancer cell lines. We also evaluated the level of survivin protein expression in the cell lines by western blot analysis (Fig. 2) which was further confirmed by qRT-PCR (Fig. 3). 

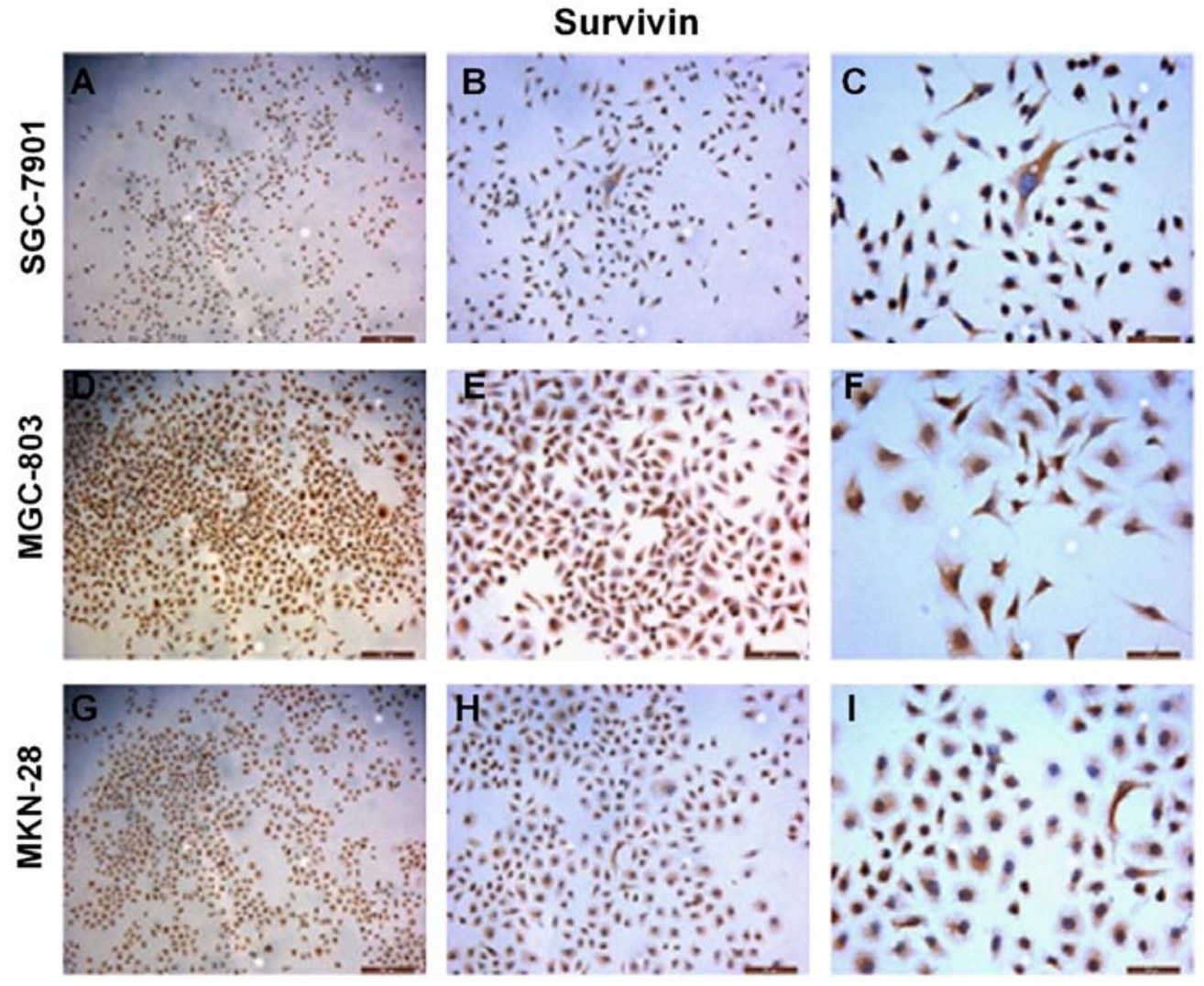

Figure 1. Immunocytochemical analysis of survivin expression in the gastric cancer cell lines. Light microscopic immunocytochemistry revealed an intense cytoplasmic presence of survivin protein. (A-C) Survivin expression in the SGC-7901 cell line at different cell resolutions. (D-F) Survivin expression in the MGC-803 cell line at different resolutions. (G-I) Strong cytoplasmic survivin expression in the MKN-28 cell line at different resolutions.

Lentiviral-mediated RNAi efficiently suppresses survivin protein and $m R N A$ expression in the gastric cancer cell lines. Lentiviral-mediated survivin shRNAs specifically knocked down the survivin expression and activity in the gastric cancer cell lines. All gastric cancer cell lines used expressed high levels of cytoplasmic survivin expression, and exhibited aggressive growth and metastatic ability. To investigate the role of survivin in gastric cancer cell growth and metastasis, we constructed the lentiviral vector with survivin shRNA and infected the cell lines. After viral infection, $>95 \%$ of the cells were GFP-positive, indicating a high efficiency of shRNA delivery. Survivin shRNA more efficiently knocked down protein expression in the cell lines as compared to survivin expression in the normal control and non-silencing group $(\mathrm{p}<0.05)$ (Fig. 2). Survivin shRNA also efficiently suppressed the survivin mRNA level as confirmed by qRT-PCR (Fig. 3).

Effects of survivin shRNA on the proliferation of SGC-7901, $M G C-803$ and $M K N-28$ cells. To determine the effects of the silencing of survivin on the proliferation of SGC-7901, MGC-803 and MKN-28 cells, we treated these cells with different doses of survivin shRNA for different periods. CCK-8 assay was then used to evaluate cell proliferation, and cell growth curves were generated. The results revealed that the gastric cancer cell lines stably transfected with survivin shRNA exhibited significantly reduced cell proliferation relative to the normal control and non-silencing group (Fig. 4A).
Survivin downregulation caused impairment of proliferation in the cells. Survivin knockdown inhibited the proliferation of the gastric cancer cell lines in vitro, indicating that the expression of survivin significantly affects the growth of gastric cancer cells.

Knockdown of survivin induces gastric cancer cell apoptosis. As shown in Fig. 4B, the percentage of apoptotic cells in the group infected with survivin shRNA was much higher than that in the control shRNA group $(\mathrm{p}<0.01)$. No significant difference in regards to apoptosis was found between the control shRNAinfected cells and the non-infected cells. These data indicated that knockdown of surviving expression induced apoptosis in the gastric cancer cells.

Lentiviral-mediated survivin shRNA significantly impairs migration and invasion of gastric cancer cells. Cell migration was evaluated in the Boyden migration assay 2 days after the gastric cancer cell lines were stably transfected with survivin shRNA or transfected with the control shRNA. Cells with motile capacity were able to migrate through the pores of the Transwell filters due to attraction to 10\% FBS in the lower chamber. Cells transfected with survivin shRNA displayed lower migratory ability compared with this ability in the nontransfected control cells (Fig. 5). The introduction of survivin shRNA into the cells, however, markedly decreased the cell migration, in comparison to the non-transfected cells. To evaluate the function of survivin shRNA on gastric cancer cell 
A
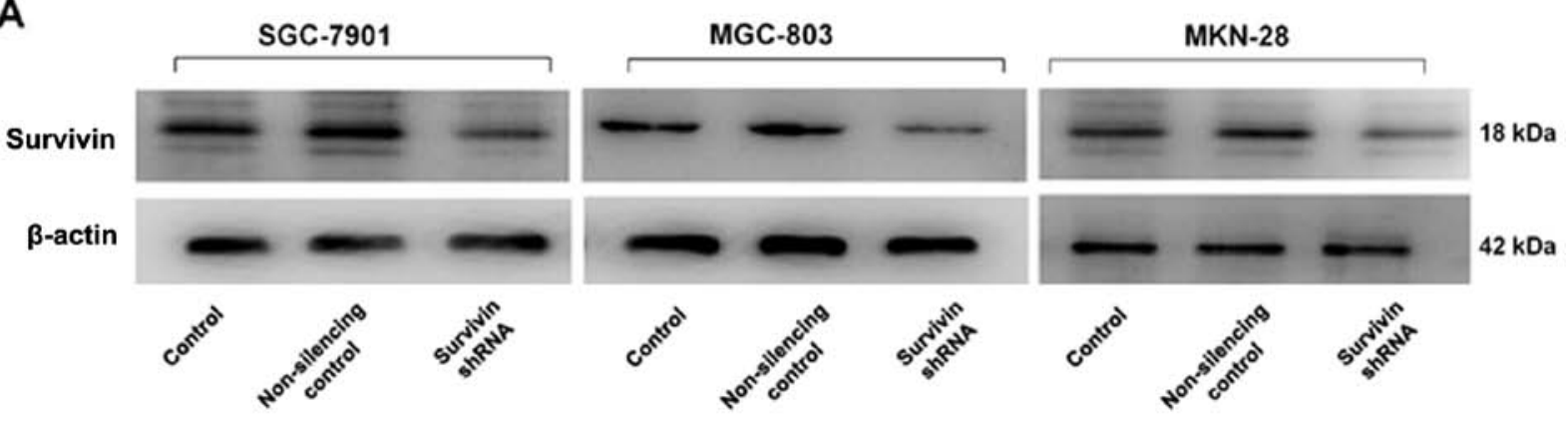

B
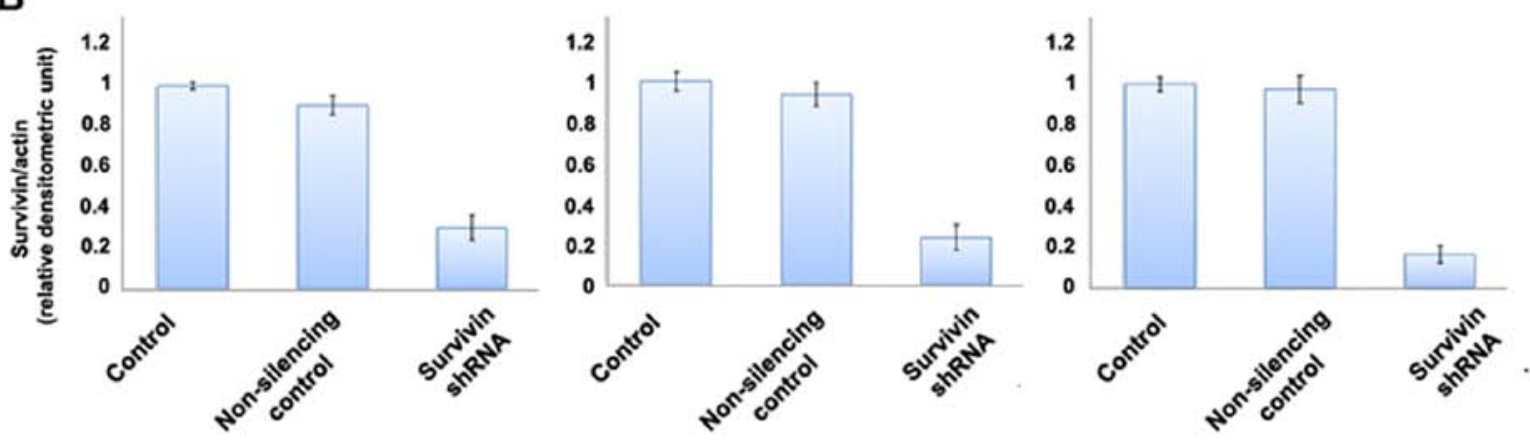

Figure 2. Gene knockdown verification at the protein level by western blot analysis. (A) Western blot analysis of survivin protein in the lysates of either the untreated (control) cells or the cells transfected with a non-silencing shRNA (scrambled sequence) or transfected with a specific survivin shRNA. $\beta$-actin expression was used as a loading control. (B) Laser densitometric analysis of protein bands was performed, and the ratio of survivin expression to $\beta$-actin expression (STMN: $\beta$-actin) was determined and normalized. Data are expressed as percent change (means \pm SD) compared with the controls and represent four independent experiments [ $\mathrm{p}<0.05$ vs. non-silencing shRNA; one-way analysis of variance (ANOVA) followed by Tukey's multiple comparison].
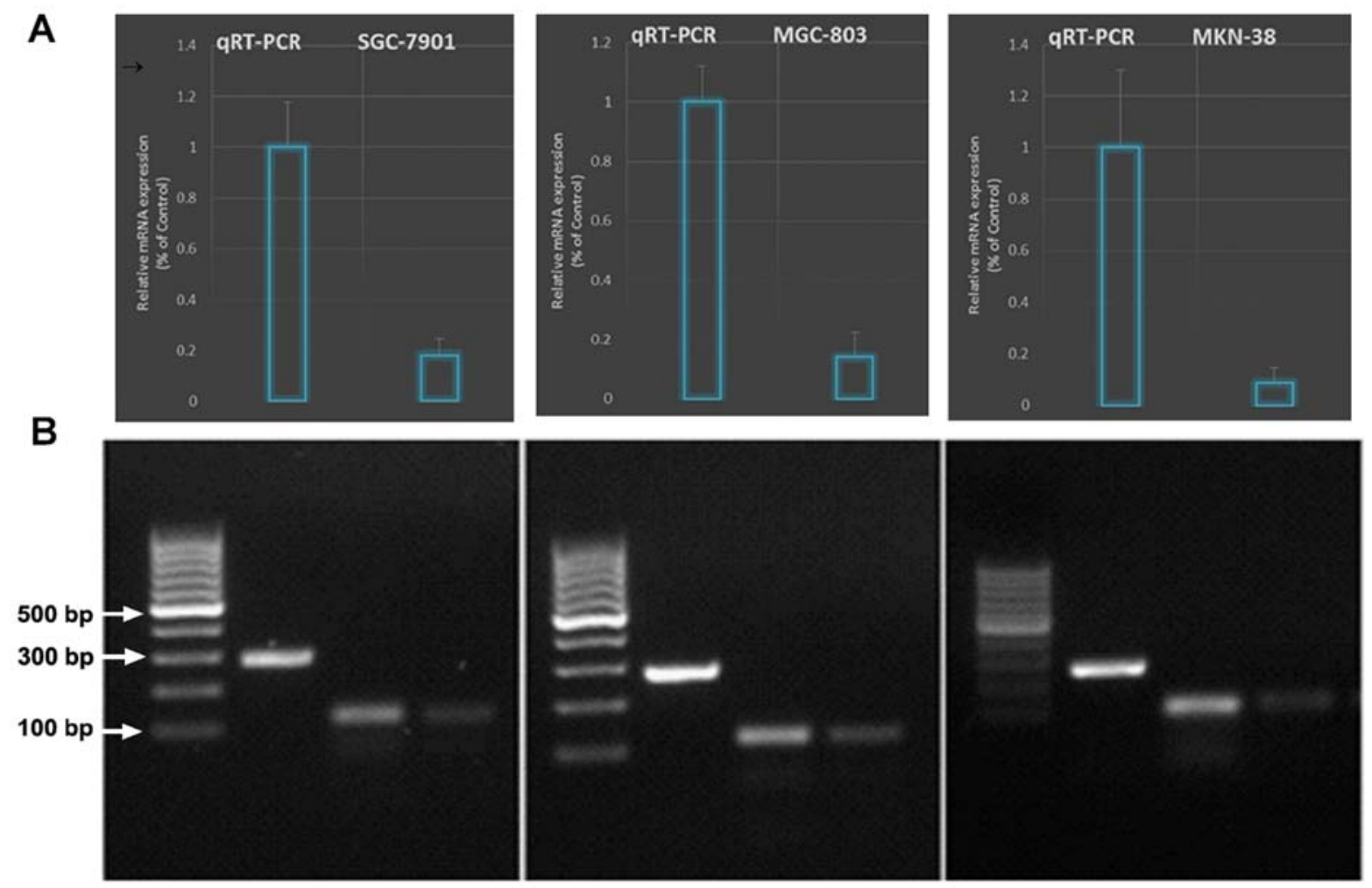

Figure 3. Gene knockdown verification at the mRNA level by qRT-PCR analysis. For a successful gene knockdown experiment, the sequences of the siRNA employed are of crucial importance. Not all siRNA sequences are able to suppress gene expression with the same efficiency. (A) For gene verification of mRNA, total RNA was isolated from the gastric cancer cell lines stably transduced with survivin shRNA or with the non-silencing shRNA and was then analyzed with real-time RT-PCR. There was no significant difference between the controls and the non-silencing shRNA (scrambled sequences). A reduction in the mRNA level of $>90 \%$ was obtained with the most efficient siRNA sequences. (B) Agarose gel electrophoresis was used to separate the DNA fragment. Data are expressed as percent change (means $\pm \mathrm{SD}$ ) compared with the controls and represent four independent experiments [p $<0.05 \mathrm{vs}$. non-silencing shRNA; one-way analysis of variance (ANOVA) followed by Tukey's multiple comparison]. 
A

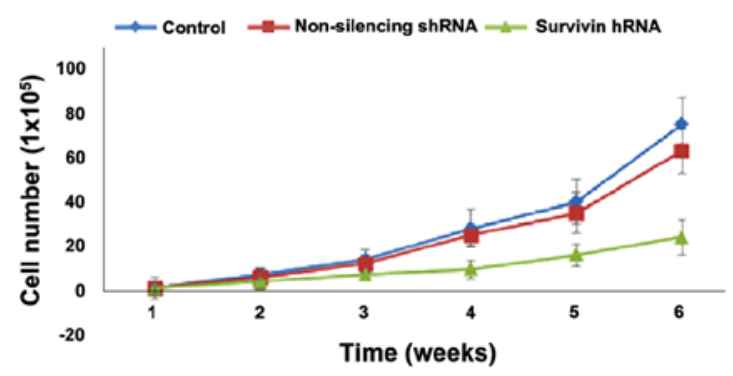

MGC-803

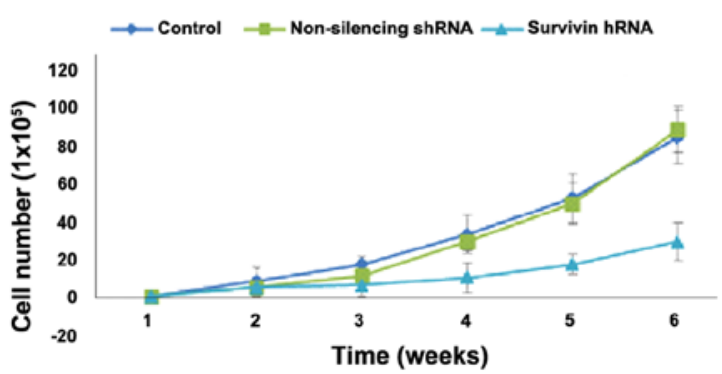

MKN-28

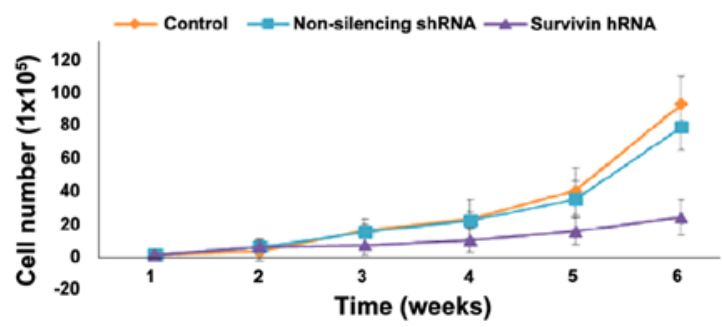

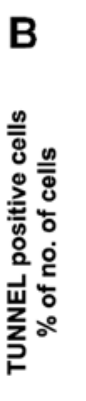
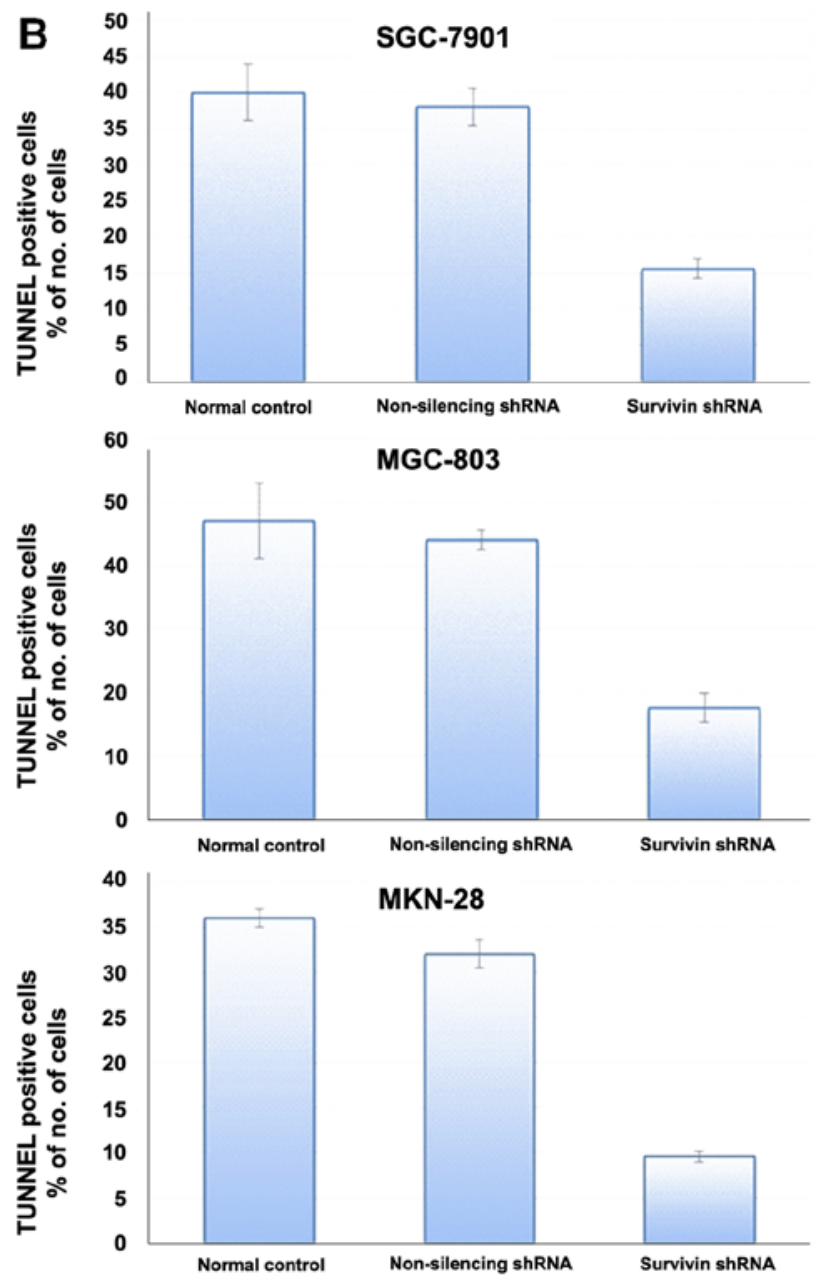

Figure 4. Effects of survivin knockdown on cell proliferation and apoptosis. (A) Gastric cancer cell lines were stably transfected with the non-silencing shRNA as a negative control or survivin shRNA. Cell proliferation was measured by CCK-8 assay. Data are expressed as percent change (means \pm SD) compared with the controls and represent six independent experiments $[\mathrm{P}<0.05$ vs. non-silencing shRNA; one-way analysis of variance (ANOVA) followed by Tukey's multiple comparison]. (B) Apoptosis was assessed by TUNEL assay. The amount of DNA fragmentation (apoptosis) was assessed by TUNEL assay in the gastric cancer cell lines stably transfected with the non-silencing shRNA as a negative control or survivin shRNA. There was no significant difference between the controls and the non-silencing shRNA. Experiments were performed in triplicate.

invasion, Matrigel invasion chambers were utilized. Silencing of survivin expression led to a significant decrease in the invasive ability of the gastric cancer cells.

In vivo studies of gastric cancer xenograft tumor models in nude mice. To further evaluate the effects of reduced survivin expression on the tumorigenic phenotype and in particular its contribution to in vivo tumor growth, gastric cancer cell lines stably transfected with the non-silencing shRNA or survivin shRNA or the untreated cells were injected into mice $\left(2 \times 10^{6}\right.$ cells in $200 \mu \mathrm{l}$ PBS) to develop a xenograft model of human gastric cancer. Tumors were measured using Vernier calipers for calculation of tumor size. Tumors derived from the survivin shRNA-transfected cells grew less rapidly as compared to the negative control $(\mathrm{p}<0.05)$. Photographic image of nude mice bearing xenograft tumors (Fig. 6A) and image of the xenografts dissected from the nude mice are shown (Fig. 6B). These results demonstrate that the in vivo tumor growth was inhibited by shRNA-mediated knockdown of survivin expression in the gastric cancer cell lines.
Tumor growth and weight and evaluation of the antitumoral effect. Tumor volume was evaluated twice a week by measuring two perpendicular diameters with calipers (Fig. 6C). Tumor volume (V) was calculated using the following equation: $\mathrm{V}=\left(\mathrm{a}^{2} \mathrm{x} b\right) / 2$ where $\mathrm{a}$ is the width of the tumor (small diameter) and $b$ the length (large diameter), both in millimeters. The mean tumor weight of the resected tumors is shown in Fig. 6D. Tumor weight was significantly reduced in the survivin shRNA knockdown xenograft tumors.

\section{Discussion}

The major aim of the present study was to investigate the effect of lentiviral-mediated knockdown of survivin on cell proliferation in vitro and tumor growth in vivo. Survivin is a recently discovered IAP that is unique for its expression in a wide range of embryonic and fetal tissues but is lowly expressed in terminally differentiated normal adult tissues. Survivin is widely implicated in processes related to tumor development and progression due to its ability to inhibit 
A
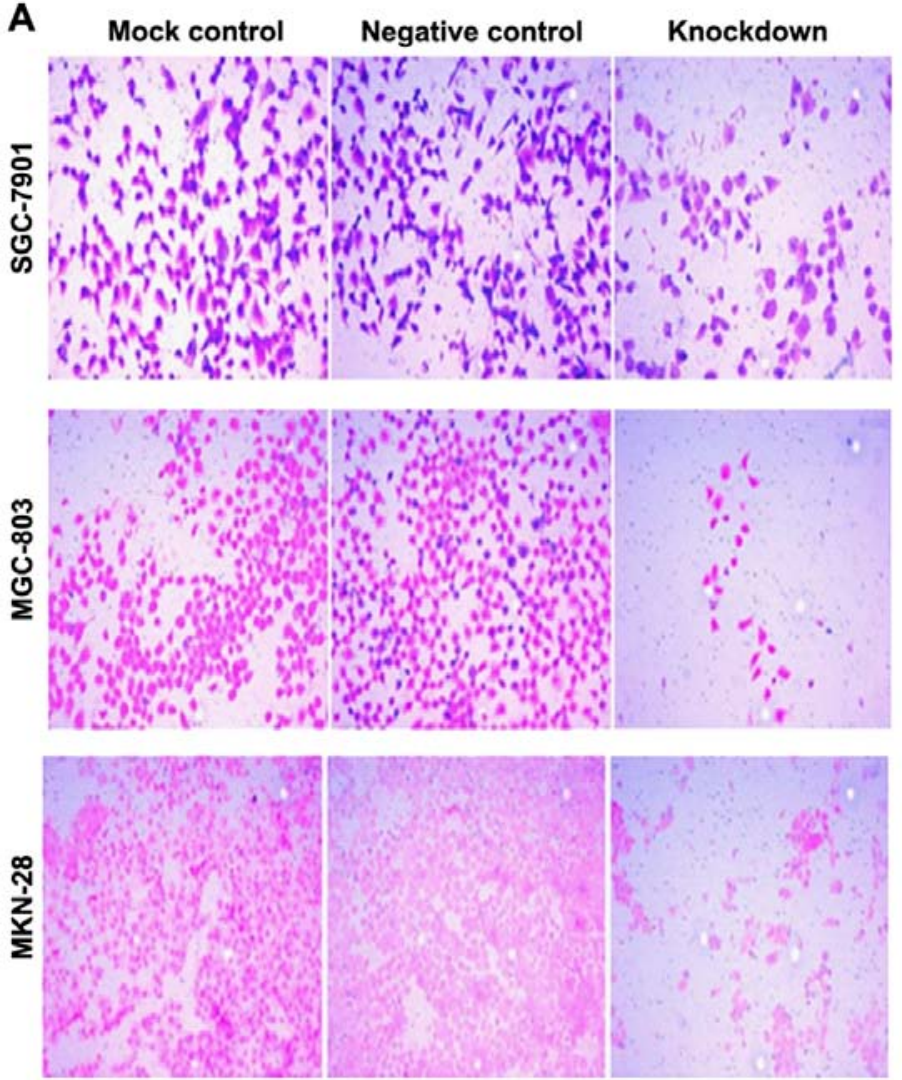

B
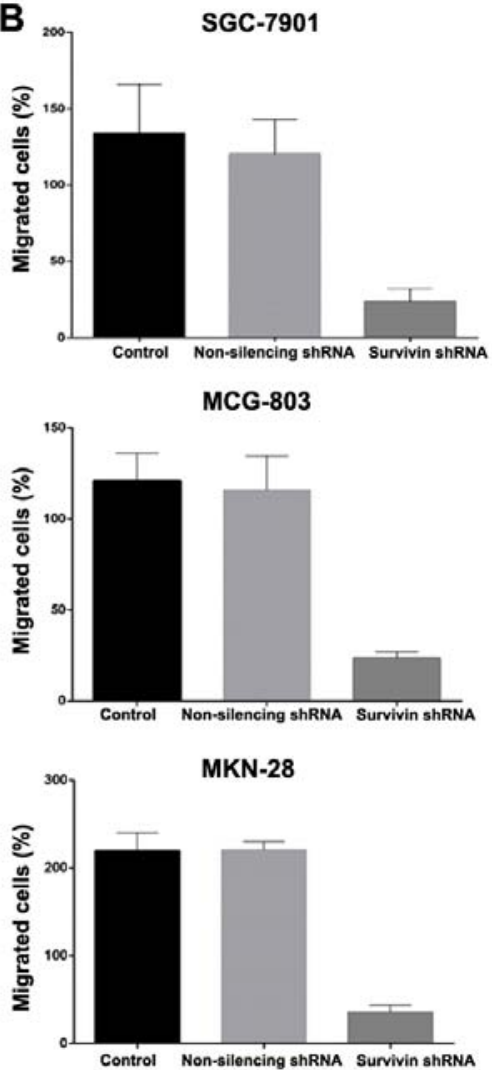

Figure 5. Effects of survivin knockdown on cell migration and invasion. Images of cell migration through the PVDF filters as examined by cell migration assay using Boyden chambers. (A) Cell migration was evaluated in the Boyden migration assay $72 \mathrm{~h}$ after gastric cancer cell lines were stably transfected with either the control or transfected with the non-silencing shRNA or survivin shRNA. (B) Percentage of migrating cells in the survivin shRNA-infected cells, the non-silencing shRNA-infected cells and the non-infected cells $(\mathrm{p}<0.05)$ vs. the control in all three gastric cancer cell lines. Data are expressed as percent change (means $\pm \mathrm{SD}$ ) compared with the controls and represent four independent experiments [p $<0.05$ vs. non-silencing shRNA; one-way analysis of variance (ANOVA) followed by Tukey's multiple comparison].

apoptosis, promote cell cycle progression, accelerate metastasis and enhance angiogenesis (24). The IAP and BCL-2 family proteins are critically important for the regulation of apoptosis (6). Survivin is also required for the maintenance of the spindle assembly checkpoint to allow proper microtubule alignment to ensure cell propagation (25). Lack of survivin during cell division causes polyploidy as well as apoptosis (26). The robust expression of survivin in gastric cancer vs. normal cells, its correlation with poor prognosis and resistance to therapy suggest that survivin is an inducible resistance factor in cancer cells and is involved in the emergence of a refractory phenotype to anticancer therapies. Similar to the conserved mechanism in the developmental process, survivin inhibition has been shown to enhance cell death (27). Various approaches have been attempted to downregulate or block survivin in cancer cells to inhibit cell survival and at the same time enhance cell death.

RNAi is a relatively new technology and holds promise for the development of therapeutic gene silencing. However, the knockdown effect of regular synthesized siRNA only lasts for a short time and does not allow the stable inhibition of target gene function. Lentiviral vectors allow efficient delivery and stable transfection of a gene of interest. In previous studies, we tested the efficacies of lentiviral vectors for shRNA delivery in cancer cells $(21,22)$. An approach using short shRNA successfully reduced survivin expression, and induced apoptosis and growth inhibition in a lymphoma cell line (28). In the present research, we used lentivirus-based vectors to silence survivin expression in gastric cancer cell lines (SGC-7901, MGC-803 and MKN-28). We found lentiviral-mediated shRNA was highly capable of stable knockdown of survivin expression in the cell lines.

Increased survivin mRNA or protein expression has previously been reported to be a prognostic indicator of tumor progression in different types of human cancer (7-12). Cytoplasmic expression of survivin is common in breast cancer and may be a useful clinical diagnostic and prognostic marker (13). Survivin expression has previously been observed in gastric cancer, yet no in vitro or in vivo data are available to date regarding the role of lentiviral-mediated knockdown of survivin expression in this cancer type. Therefore, we quantified the expression levels of survivin in three different gastric cancer cell lines and downregulated the survivin expression using shRNA. The results of ICC, western blot analysis and qRT-PCR revealed that survivin expression was upregulated in all three gastric cancer cell lines. We constructed a lentiviral vector against survivin and stably transduced gastric cell lines with survivin shRNA. The results showed that expression of survivin protein in all three cell lines transfected with survivin shRNA was reduced compared with that in the non-silencing control cells. Furthermore, the proteins extracted from the cell lines were analyzed with western blot 

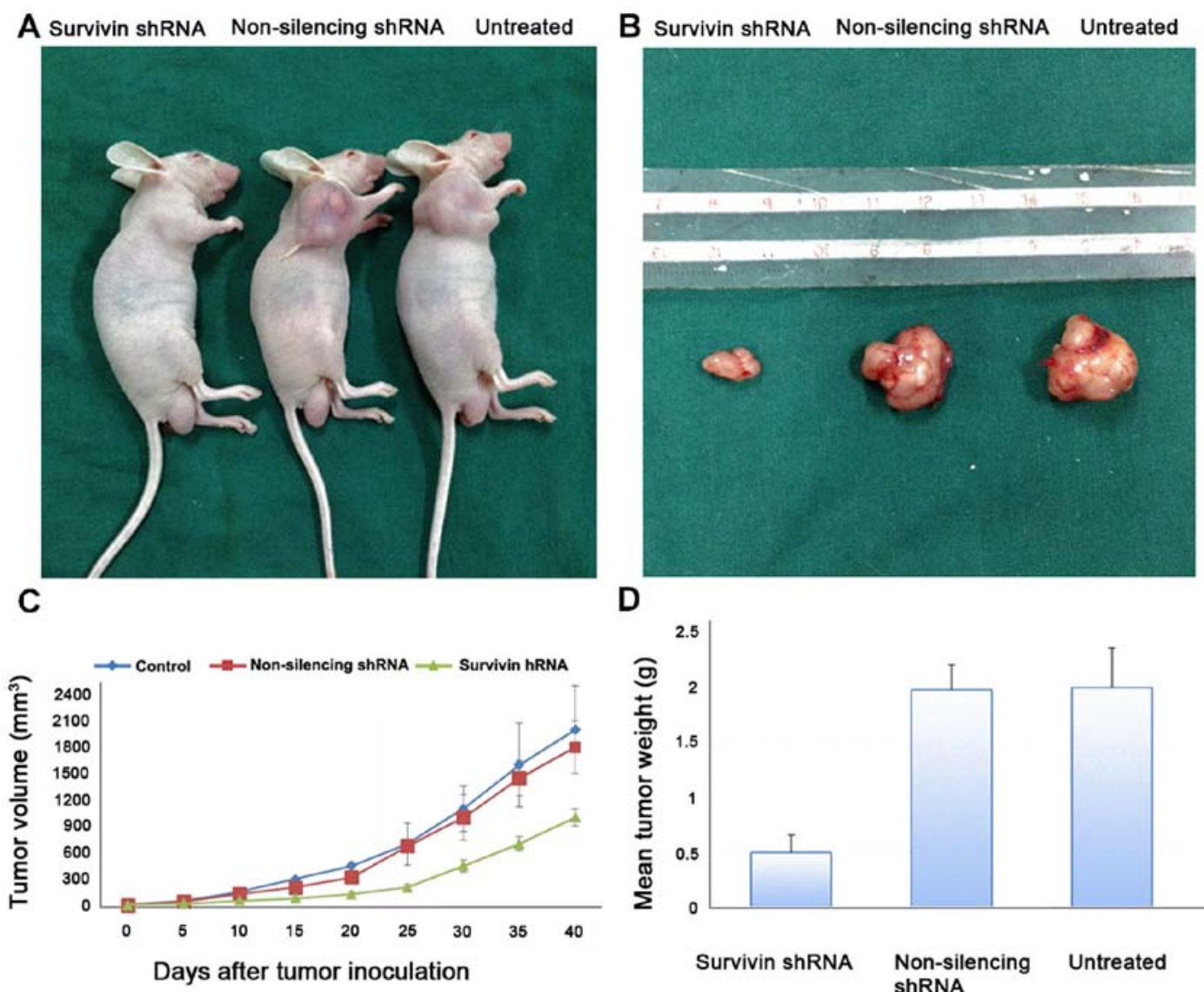

D

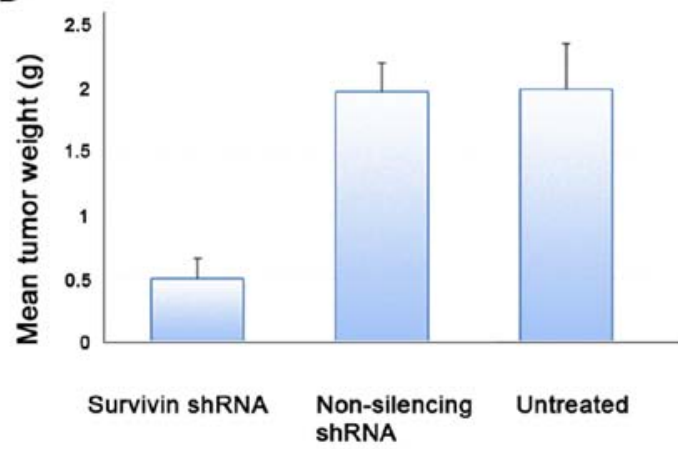

Figure 6. Xenograft tumor models in nude mice. Gastric cancer cells either untreated or transfected with the non-silencing shRNA (scrambled sequence) as a negative control or transfected with survivin shRNA were xenografted subcutaneously in the BALB/c-nu/nu male mice. (A) Representative photographic image of nude mice bearing xenograft tumors. (B) Images of xenografts dissected from the nude mice 7 weeks after subcutaneous inoculation. Suppression of the growth of tumors derived from cancer cells transfected with survivin shRNA was noted when compared with the tumors derived from the cells transfected with the non-silencing shRNA or the untreated cells. (C) Tumor volume curve showing the growth of the tumors and (D) graph showing the mean tumor weight of the resected tumor. Tumor mass (xenograft) volume was measured at least twice a week from week 3 to 7 . Data are expressed as percent change (means \pm SD) compared with the controls and represent four independent experiments [ $p<0.05$ vs. non-silencing shRNA; one-way analysis of variance (ANOVA) followed by Tukey's multiple comparison]

analysis. The transfection results were stable, persistence and consistent with the mRNA levels. The duration of gene downregulation was better than the transient cell transfection. The present study provides in vitro and in vivo evidence for a role of survivin shRNA in the pathogenesis and progression of gastric cancer.

In the present study, we investigated the function of survivin in gastric cancer and demonstrated that silencing of survivin by RNAi led to reduced proliferation and migration in gastric cancer SGC-7901, MGC-803 and MKN-28 cell lines. The findings indicate that survivin may take part in the development, progression and metastasis of gastric cancer or prognosis of the patients by regulating proliferation and migration of gastric cancer cells. Researcher has demonstrated that simultaneous inhibition of survivin and XIAP by siRNA significantly reduced cell proliferation and increased caspase$3 / 7$ activity in pancreatic carcinoma cells (29). Inhibition of apoptosis is believed to be through direct binding to caspase-3 and -7 , preventing their activation (30). The effect of survivin knockdown by siRNA on the proliferation of two human cancer cell lines has also been reported (31). At least one mechanism of survivin involves the suppression of default apoptosis in the G2/M phase (14).
Furthermore, in the xenograft model, survivin shRNA potently inhibited the in vivo growth of gastric cancer xenografts, resulting in tumor regression, while the mouse tumors derived from the untreated cells grew rapidly and aggressively. Tumor growth was significantly decreased in the survivin shRNA transfection groups as compared with the control groups. Various therapeutics are associated with toxic side effects such as weight loss. These systemic toxicities may be related with higher morbidity and a lower response rate, resulting in poorer survival. However, in our animal model, weight loss and toxic effects of treatment were not obvious (data not shown). In the present study, the measured body weights of mice had no differences (data not shown) and there were no significant pathologic findings from tissue obtained from the shRNA-treated group. For these reasons, shRNA targeting survivin can be used in gastric cancer therapy without systemic toxic effects in vivo. In the present study, despite an effective reduction in tumor size, we observed marked tumor regression, with tumors displaying extensive areas of necrotic tissue and a significant decrease in the number of blood vessels (data not shown).

In conclusion, these findings indicate for the first time that targeting cytoplasmic survivin expression utilizing 
lentiviral survivin shRNA in gastric cancer cell lines reduced the progression of cancer. We also showed that the lentiviral vector targeting survivin expression blocked the growth and induced cell death in the gastric carcinoma cell lines in vitro and in vivo.

\section{References}

1. Jemal A, Bray F, Center MM, Ferlay J, Ward E and Forman D: Global cancer statistics. CA Cancer J Clin 61: 69-90, 2011.

2. Siegel R, Naishadham D and Jemal A: Cancer statistics, 2013. CA Cancer J Clin 63: 11-30, 2013.

3. Yamamoto H, Ngan CY and Monden M: Cancer cells survive with survivin. Cancer Sci 99: 1709-1714, 2008.

4. Ambrosini G, Adida C and Altieri DC: A novel anti-apoptosis gene, survivin, expressed in cancer and lymphoma. Nat Med 3: 917-921, 1997.

5. Li F, Ambrosini G, Chu EY, Plescia J, Tognin S, Marchisio PC and Altieri DC: Control of apoptosis and mitotic spindle checkpoint by survivin 396: 580-584, 1998.

6. Jäättelä M: Escaping cell death: Survival proteins in cancer. Exp Cell Res 248: 30-43, 1999.

7. Adida C, Berrebi D, Peuchmaur M, Reyes-Mugica M and Altieri DC: Anti-apoptosis gene, survivin, and prognosis of neuroblastoma. Lancet 351: 882-883, 1998.

8. Kawasaki H, Altieri DC, Lu C-D, Toyoda M, Tenjo T and Tanigawa N: Inhibition of apoptosis by survivin predicts shorter survival rates in colorectal cancer. Cancer Res 58: 5071-5074, 1998.

9. Monzó M, Rosell R, Felip E, Astudillo J, Sánchez JJ, Maestre J, Martín C, Font A, Barnadas A and Abad A: A novel anti-apoptosis gene: Re-expression of survivin messenger RNA as a prognosis marker in non-small-cell lung cancers. J Clin Oncol 17: 2100-2104, 1999.

10. Swana HS, Grossman D, Anthony JN, Weiss RM and Altieri DC: Tumor content of the antiapoptosis molecule survivin and recurrence of bladder cancer. N Engl J Med 341: 452-453, 1999

11. Sarela AI, Macadam RC, Farmery SM, Markham AF and Guillou PJ: Expression of the antiapoptosis gene, survivin, predicts death from recurrent colorectal carcinoma. Gut 46 : 645-650, 2000.

12. Kato J, Kuwabara Y, Mitani M, Shinoda N, Sato A, Toyama T, Mitsui A, Nishiwaki T, Moriyama S, Kudo J, et al: Expression of survivin in esophageal cancer: Correlation with the prognosis and response to chemotherapy. Int J Cancer 95: 92-95, 2001.

13. Sohn DM, Kim SY, Baek MJ, Lim CW, Lee MH, Cho MS and Kim TY: Expression of survivin and clinical correlation in patients with breast cancer. Biomed Pharmacother 60: 289-292, 2006.

14. Shin S, Sung B-J, Cho Y-S, Kim HJ, Ha NC, Hwang JI, Chung CW, Jung YK and Oh BH: An anti-apoptotic protein human survivin is a direct inhibitor of caspase-3 and -7. Biochemistry 40: $1117-1123,2001$.

15. Adamkov M, Kajo K, Vybohova D, Krajcovic J, Stuller F and Rajcani J: Correlations of survivin expression with clinicomorphological parameters and hormonal receptor status in breast ductal carcinoma. Neoplasma 59: 30-37, 2012.
16. Sui L, Dong Y, Ohno M, Watanabe Y, Sugimoto K and Tokuda M: Survivin expression and its correlation with cell proliferation and prognosis in epithelial ovarian tumors. Int J Oncol 21: 315-320, 2002.

17. Tanaka K, Iwamoto S, Gon G, Nohara T, Iwamoto M and Tanigawa N: Expression of survivin and its relationship to loss of apoptosis in breast carcinomas. Clin Cancer Res 6: 127-134, 2000.

18. Zaffaroni N, Pennati M, Colella G, Perego P, Supino R, Gatti L, Pilotti S, Zunino F and Daidone MG: Expression of the anti-apoptotic gene survivin correlates with taxol resistance in human ovarian cancer. Cell Mol Life Sci 59: 1406-1412, 2002.

19. Mehrotra S, Languino LR, Raskett CM, Mercurio AM, Dohi T and Altieri DC: IAP regulation of metastasis. Cancer Cell 17: 53-64, 2010.

20. Yano J, Hirabayashi K, Nakagawa S, Yamaguchi T, Nogawa M, Kashimori I, Naito H, Kitagawa H, Ishiyama K, Ohgi T, et al: Antitumor activity of small interfering RNA/cationic liposome complex in mouse models of cancer. Clin Cancer Res 10: 7721-7726, 2004

21. Akhtar J, Wang Z, Yu C and Zhang ZP: Effectiveness of local injection of lentivirus-delivered stathmin 1 and stathmin1 shRNA in human gastric cancer xenograft mouse. J Gastroenterol Hepatol 29: 1685-1691, 2014

22. Akhtar J, Wang Z, Zhang ZP and Bi MM: Lentiviral-mediated RNA interference targeting stathmin 1 gene in human gastric cancer cells inhibits proliferation in vitro and tumor growth in vivo. J Transl Med 11: 212, 2013.

23. Chawla-Sarkar M, Bae SI, Reu FJ, Jacobs BS, Lindner DJ and Borden EC: Downregulation of Bcl-2, FLIP or IAPs (XIAP and survivin) by siRNAs sensitizes resistant melanoma cells to Apo2L/TRAIL-induced apoptosis. Cell Death Differ 11: 915-923, 2004.

24. Altieri DC: Survivin, cancer networks and pathway-directed drug discovery. Nat Rev Cancer 8: 61-70, 2008

25. Lens SMA, Wolthuis RMF, Klompmaker R, Kauw J, Agami R, Brummelkamp T, Kops G and Medema RH: Survivin is required for a sustained spindle checkpoint arrest in response to lack of tension. EMBO J 22: 2934-2947, 2003.

26. Yang D, Welm A and Bishop JM: Cell division and cell survival in the absence of survivin. Proc Natl Acad Sci USA 101: 15100-15105, 2004.

27. Altieri DC: Validating survivin as a cancer therapeutic target. Nat Rev Cancer 3: 46-54, 2003.

28. Gu CM, Zhu YK, Ma YH, Zhang M, Liao B, Wu HY and Lin HL: Knockdown of survivin gene by vector-based short hairpin RNA technique induces apoptosis and growth inhibition in Burkitt's lymphoma Raji cell line. Neoplasma 53: 206-212, 2006.

29. Yang J, Ouyang J, Ouyang L, Ouyang L and Chen Y: Inhibition of cell proliferation and increase of chemosensitivity by simultaneous knockdown of XIAP and survivin in pancreatic carcinoma cells. Oncol Res 21: 43-50, 2013.

30. Tamm I, Wang Y, Sausville E, Scudiero DA, Vigna N, Oltersdorf T and Reed JC: IAP-family protein survivin inhibits caspase activity and apoptosis induced by Fas (CD95), Bax, caspases, and anticancer drugs. Cancer Res 58: 5315-5320, 1998.

31. Guan HT, Xue XH, Wang XJ, Li A and Qin ZY: Knockdown of survivin expression by small interfering RNA suppresses proliferation of two human cancer cell lines. Chin Med Sci J 21: $115-119,2006$ 